\title{
A Tiny Melanoma: The Beginning of a Life
}

\author{
Aimilios Lallas ${ }^{1}$, Chryssoula Papageorgiou ${ }^{1}$, Christina Nikolaidou ${ }^{2}, Z^{2}$ ee Apalla ${ }^{3}$
}

1 First Department of Dermatology, Aristotle University, Thessaloniki, Greece

2 Department of Histopathology, Hippokration General Hospital, Thessaloniki, Greece

3 State Clinic of Dermatology, Hospital for Skin and Venereal Diseases, Thessaloniki, Greece

Key words: melanoma in situ, dermoscopy, diagnosis

Citation: Lallas A, Papageorgiou C, Nikolaidou C, Apalla Z. A tiny melanoma: the beginning of a life. Dermatol Pract Concept. 2019;9(3):207-208. DOI: https://doi.org/10.5826/dpc.0903a06

Accepted: May 2, 2019; Published: July 31, 2019

Copyright: $@ 2019$ Lallas et al. This is an open-access article distributed under the terms of the Creative Commons Attribution License, which permits unrestricted use, distribution, and reproduction in any medium, provided the original author and source are credited.

Funding: None.

Competing interests: The authors have no conflicts of interest to disclose.

Authorship: All authors have contributed significantly to this publication.

Corresponding author: Aimilios Lallas, MD, PhD, First Department of Dermatology, Aristotle University, 124 Delfon Street, Thessaloniki, Greece.Email: emlallas@gmail.com

\section{Case Presentation}

A 54-year-old man without personal or family history of melanoma was examined during a skin cancer screening campaign. The clinical examination revealed numerous nevi, angiomas, and seborrheic keratoses, all smaller than $5 \mathrm{~mm}$ and without morphological atypia (Figure 1A).

Surprisingly, the dermoscopic examination of a 2-mmsized lesion (Figure 1B) on his thorax revealed irregularly shaped hyperpigmented areas, a feature recently proposed to typify melanoma in situ (Figure 1C) [1]. The lesion was excised with a 4-mm punch, and histopathology revealed an intraepidermal lentiginous and focally nested melanocytic proliferation with moderate to severe cytological atypia and pagetoid spread. The nests were variable in size and contained melanocytes with hyperchromatic cytoplasm. Immunostaining revealed hyperplasia and asymmetric distribution of intraepidermal melanocytes and confirmed the pagetoid spread. A diagnosis of melanoma in situ was rendered.

\section{Teaching Point}

Dermoscopy was introduced as a second-level method to further evaluate clinically preselected suspicious lesions. The size threshold for a lesion to be considered suspicious is $6 \mathrm{~mm}$. Our case suggests that no size threshold should be used for dermoscopic examination, since dermoscopy has the potential to uncover even smaller melanomas and help to eradicate a potentially life-threatening tumor with a minimally invasive intervention.

\section{Reference}

1. Lallas A, Longo C, Manfredini M, et al. Accuracy of dermoscopic criteria for the diagnosis of melanoma in situ. JAMA Dermatol. 2018;154(4):414-419. 


\section{A}

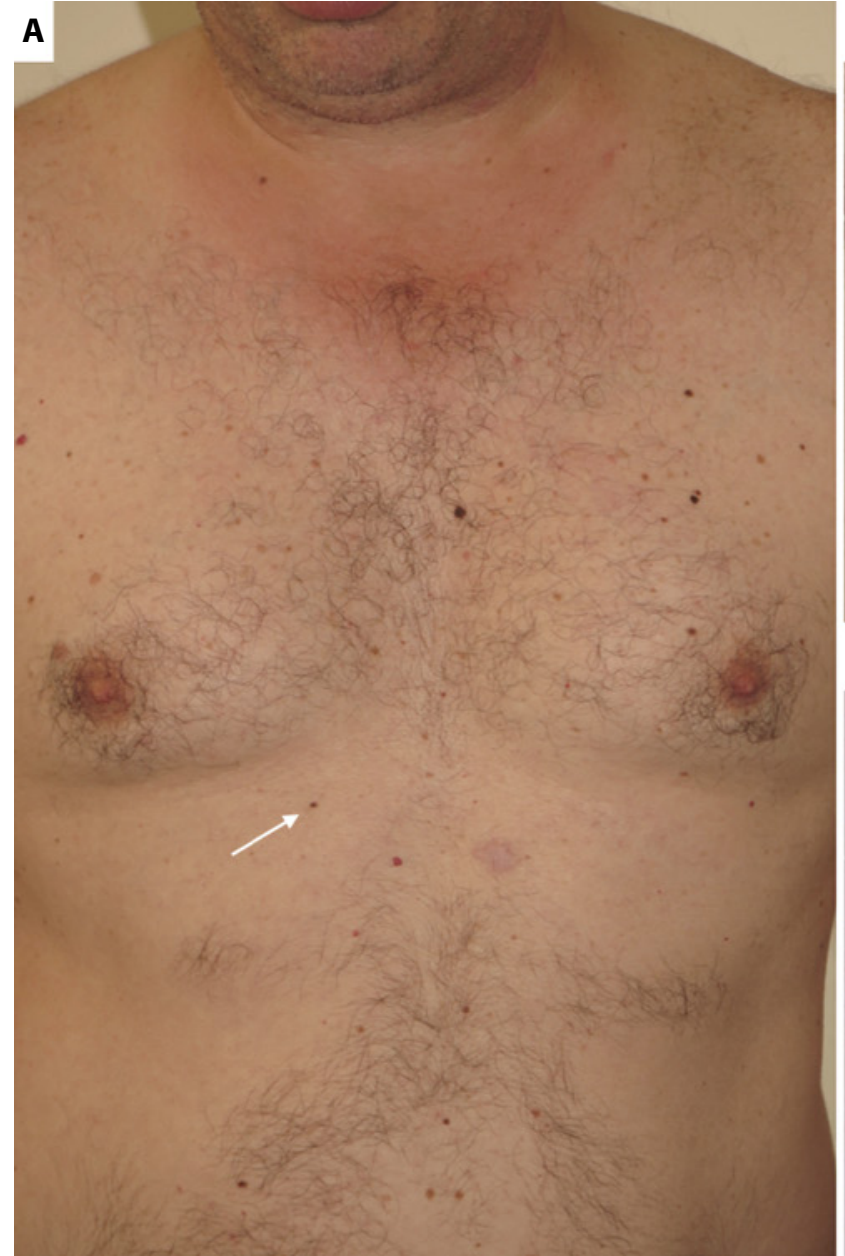

B

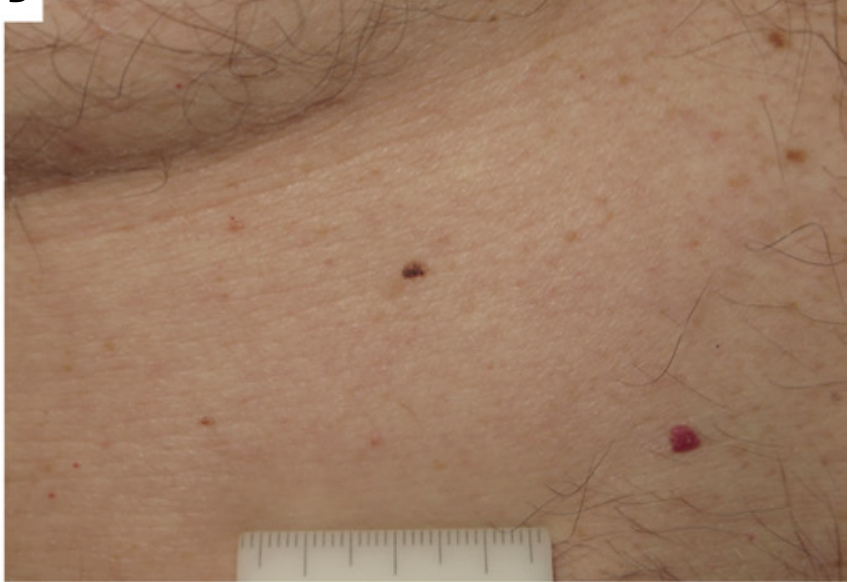

C

Figure 1. A tiny pigmented skin lesion on the chest, barely seen macroscopically (A). Close inspection reveals a 2-mm diameter brown macule (B). Dermoscopy reveals irregularly shaped hyperpigmented areas, raising the suspicioun of an early melanoma, which was histopathologically confirmed (C). [Copyright: (2019 Lallas et al.] 